\title{
Robust Controller for Constrained Relative Motion Maneuvering with Disturbance Rejection
}

\author{
Morgan Baldwin, R. Scott Erwin† and Ilya Kolmanovsky ${ }^{\ddagger}$
}

\begin{abstract}
The development and demonstration in simulations of a computationally fast control framework for robust spacecraft relative motion maneuvering with constraints is presented. This framework is capable of handling bounded disturbances (e.g. thrust errors, air drag, solar pressure, etc.) while guaranteeing that specified constraints are satisfied and the target set is reached for all disturbances with values satisfying specified bounds. The maneuver computations reduce to solving a quadratic programming problem (or a linear programming problem for a less flexible formulation) at the initial time instant to find a feasible maneuver path, and then generating feedback corrections along the maneuver path based on a simple constant gain linear feedback. Simulation results for two and three dimensional spacecraft relative motion are given.
\end{abstract}

\section{Introduction}

Autonomous spacecraft rendezvous and relative motion maneuvers are among the most important and difficult elements of spacecraft missions. One of the most prevalant examples of a relative motion maneuver is a space vehicle docking with the International Space Station (ISS). Traditionally, relative motion maneuvers are performed using open-loop maneuver planning and ad hoc error correction. The motivation for the development of a robust constrained controller is to enhance traditional open-loop spacecraft relative motion maneuver planning with compensation for thruster uncertainties, model uncertainties, and air drag disturbances. Using this framework and a spacecraft relative motion model, a maneuver planner can guarantee that for the maneuver found, the specified constraints will be satisfied for all disturbances with values in a given set and the spacecraft will reach a prescribed target set within a specified time interval.

Several methods for attitude control with disturbance rejection have been developed. ${ }^{1-3}$ A sliding mode control was shown to be effective for disturbance rejection in Ref. 2. Large angle maneuvers were handled using a nonlinear $H_{\infty}$ controller, which was designed to achieve stability and disturbance rejection. ${ }^{1}$ In Ref. 3 , a nonlinear control law for tracking attitude trajectories was developed with almost global stabilizability and capability to handle torque disturbances.

In recent years, several trajectory generation schemes for relative motion maneuvering with constraints have been developed. In Ref. 4, a rigorous methodology for handling highly constrained trajectories without ground support is developed. Artificial potential function guidance was shown to handle constraints and obstacles for relative motion maneuvering in Ref. 5. A method encompassing keep out zones while minimizing fuel expenditure is given in Ref. 6. The Inverse Dynamics in the Virtual Domain approach presented in Ref. 7 was shown to minimize fuel consumption and handle path constraints. However, none of these approaches handle disturbance rejection and the constraints in the unique way the algorithm presented in this work does.

The goal of this work is to develop a control method for relative motion maneuvering that steers spacecraft state trajectories to a terminal set with the specified state and control constraints enforced in the presence of bounded disturbances. The approach presented involves solving a linear programming (LP) or quadratic programming (QP) problem at the initial time instant to find optimized state and control sequences subject to tightened constraints and the condition that the spacecraft reaches a constrained admissible invariant

\footnotetext{
*Research Aerospace Engineer, Space Vehicles Directorate, Air Force Research Laboratory, Kirtland Air Force Base, NM.

${ }^{\dagger}$ Principal Research Engineer, Space Vehicles Directorate, Air Force Research Laboratory, Kirtland Air Force Base, NM.

$\ddagger$ Professor, Department of Aerospace Engineering, The University of Michigan, Ann Arbor, MI. The research of the third author was supported in part by NSF Award Number 1130160 and in part by ASEE Summer Faculty Fellow Program
} 
set of an unconstrained Linear Quadratic (LQ) controller in a specified number of steps. As the maneuver progresses, the state and control sequences are iteratively updated based on the LQ gain feedback of the difference between the expected state and an actual realized state. No on-board optimization needs to be performed while executing the maneuver beyond the initial time instant. The optimization problem solved at the initial time is a linear or a quadratic programming problem that can be solved either on-board of the spacecraft or off-board of the spacecraft and uplinked to the spacecraft. In this paper, differently from Ref. 8, a quadratic programming formulation is explored. The method proposed can be modified to include different cost functions depending on the purpose of the maneuver. For example, an emphasis may be placed on fuel expenditure versus attitude control capability of the vehicle. The details of the theory are reported in Refs. 9, 8, where the application of this approach to roll angle control for ships with fin stabilizers is reported.

The paper is organized as follows. The algorithm for the robust controller is presented in Section II followed by the problem formulation in Section III. Within Section III, the models, constraints, nominal controller design, and QP formulation are given. Finally, the simulation results are presented in Section IV.

\section{Algorithm}

The following summary of the algorithm is from Ref. 8. Consider a linear, time-invariant, discrete-time system

$$
x(k+1)=A x(k)+B u(k)+w(k),
$$

where $x \in \mathbb{R}^{n}$ is the state, $u \in \mathbb{R}^{m}$ is the control, and $w \in \mathbb{R}^{n}$ is the disturbance. The state and control are subject to hard constraints

$$
(u, x) \in \Omega \subset U \times X \text { and } w \in W,
$$

where $U$ and $W$ are polytopes and $X$ is a polyhedron. The target set $X_{t}$ is a polytope and is defined as

$$
X_{t}=\left\{x \in H^{n} \mid Y x \leq q\right\}, \quad Y \in H^{r \times n}, \quad q \in \mathbb{R}^{r} .
$$

Also, we assume that there exists a state feedback controller with the gain $K$ such that the closed-loop system

$$
x(k+1)=A_{K} x(k)+B w \quad \text { where } \quad A_{K}=A+B K
$$

is exponentially stable and the minimal robust invariant set for Eq. (2) is contained in the target set in the presence of bounded disturbances. The objective is to determine the control $u$ that steers the trajectory to the target set.

Given an initial state $x \in X$, the following control and state sequences

$$
\begin{aligned}
& \mathbf{u}^{*}(x)=\left\{u_{0}^{*}(x), u_{1}^{*}(x), \ldots, u_{N}^{*}(x)\right\}, \\
& \mathbf{x}^{*}(x)=\left\{x_{0}^{*}(x), x_{1}^{*}(x), \ldots, x_{N}^{*}(x)\right\}
\end{aligned}
$$

are feasible if they satisfy the set of constraints $C(x)$ defined as

$$
\begin{aligned}
x_{0}^{*}(x) & =x, \\
x_{i+1}^{*}(x) & =A x_{i}^{*}(x)+B u_{i}^{*}(x), \quad i=0, \ldots, N-1, \\
\Omega_{0} & =\Omega, \\
\Omega_{i+1} & =\Omega_{i} \sim\left[\begin{array}{ll}
K^{T} & I
\end{array}\right]^{T} A_{k}^{i} B W, \quad i=0, \ldots, N-1, \\
\left(u_{i}^{*}(x), x_{i}^{*}(x)\right) & \in \Omega_{i}, \quad i=0, \ldots, N-1, \\
x_{N}^{*} & \in X_{f},
\end{aligned}
$$

where $X_{f}$ is a robust invariant set for Eq. (2) with $w \in A_{K}^{N} W$. The feasible initial control and state sequences are determined by finding a point inside the polyhedron defined by $C(x)$. This point can be determined by solving a linear or a quadratic programming problem. At each time step, the feasible control sequence $\mathbf{u}^{*}(x(k))$ is constructed using the previous step's feasible control and state sequence or $\mathbf{u}^{*}(x(k-1))$ and $\mathbf{x}^{*}(x(k-1))$ where $x(k)$ is the observed state at step $k$ :

$$
\begin{aligned}
u_{i}^{*}(x(k)) & =u_{i+1}^{*}(x(k-1))+K\left(x_{i}^{*}(x(k))-x_{i+1}^{*}(x(k-1))\right) \quad i=0, \ldots, N-2, \\
u_{N-1}^{*}(x(k)) & =K x_{N-1}^{*}(x(k)) .
\end{aligned}
$$


The state sequence is updated at each step using

$$
\begin{aligned}
x_{0}^{*}(x(k)) & =x(k), \\
x_{i+1}^{*}(x(k)) & =A x_{i}^{*}(x(k))+B u_{i}^{*}(x(k)) \quad i=0, \ldots, N-1 .
\end{aligned}
$$

The first element of the feasible control sequence is applied at each step, yielding the control law

$$
u(k)=\kappa_{N}^{*}(x(k))=u_{0}^{*}(x(k)) .
$$

The steps of the algorithm are as follows:

1. At step $k=0$, find $\mathbf{u}^{*}(x(0))$ and $\mathbf{x}^{*}(x(0))$ that satisfy $C(x(0))$.

2. At step $k>0$ and given $\mathbf{u}^{*}(x(k-1))$ and $\mathbf{x}^{*}(x(k-1))$, observe $x(k)$ and calculate $\mathbf{u}^{*}(x(k))$ and $\mathbf{x}^{*}(x(k))$ using Eqs. (6) and (9):

(a) Set $i=0$ and $\mathbf{x}_{i}^{*}(x(k))=x(k)$.

(b) Set $u_{i}^{*}(x(k))=u_{i+1}^{*}(x(k-1))+K\left(x_{i}^{*}(x(k))-x_{i+1}^{*}(x(k-1))\right)$ if $i \neq N-1$. Otherwise, set $u_{i}^{*}(x(k))=K x_{i}^{*}(x(k))$.

(c) Set $x_{i+1}^{*}(x(k))=A x_{i}^{*}(x(k))+B u_{i}^{*}(x(k))$.

(d) If $i=N-1$, terminate. Otherwise, set $i=i+1$ and go to $2 \mathrm{~b}$.

3. Apply the first element of the feasible control sequence $\mathbf{u}^{*}(x(k))$.

Ref. 8 shows that the proposed controller steers state trajectories to a robust invariant set in which the states and the linear feedback control satisfy the constraints, the imposed state and control constraints are satisfied, and convergence to the target set is guaranteed after $N$ steps.

\section{Problem formulation}

For this work, a controller is designed to guarantee a chaser vehicle will dock with a target vehicle given constraints on both the state and control of the vehicle while compensating for disturbances along the trajectory. The disturbances along the trajectory may represent model and thruster uncertainties and air drag or solar pressure disturbances. The model used in this work is presented first. Second, the state and control constraints are defined. Then the nominal controller design is presented and finally the complete QP problem setup is given.

\section{A. Model}

In relative motion problems, an approaching spacecraft maneuvers close to a non-maneuvering target spacecraft in a nominal orbit. The target spacecraft is assumed to be at the origin of the Hill's frame. The equations of motion for the approaching spacecraft around a target spacecraft depend nonlinearly on the orbital radius. For circular orbits, the relative motion equations can be reduced to the well known linear time-invariant Hill-Clohessy-Wiltshire (HCW) equations (see Ref. 10). Both the two-dimensional and three-dimensional models for relative motion are given.

The relative position vector of the approaching spacecraft with respect to the target spacecraft is expressed as

$$
\delta \vec{r}=\delta x \hat{\imath}+\delta y \hat{\jmath}+\delta z \hat{k}
$$

where $\delta x, \delta y$ and $\delta z$ are the components of the position vector of the approaching spacecraft relative to the target spacecraft. The position vector of the approaching spacecraft with respect to the center of the Earth is thus given by $\vec{R}=\vec{R}_{0}+\delta \vec{r}=\left(R_{0}+\delta x\right) \hat{\imath}+\delta y \hat{\jmath}+\delta z \hat{k}$, where $\vec{R}_{0}$ is the position vehicle of the target spacecraft. The nonlinear equations of motion for the approaching spacecraft can be expressed in vector form as

$$
\ddot{\vec{R}}=-\mu \frac{\vec{R}}{R^{3}}+\frac{1}{m_{c}} \vec{F},
$$

where $\vec{F}$ reflects the vector of forces applied to the approaching spacecraft (excluding ideal gravity), $R=|\vec{R}|$, $m_{c}$ is the mass of the spacecraft, and $\mu$ is the gravitational constant. 
For $\delta r \ll R$, the linearized $\mathrm{HCW}$ equations ${ }^{10}$ approximate the relative motion of the approaching spacecraft as

$$
\begin{aligned}
\delta \ddot{x}-3 n^{2} \delta x-2 n \delta \dot{y} & =\frac{F_{x}}{m_{c}}, \\
\delta \ddot{y}+2 n \delta \dot{x} & =\frac{F_{y}}{m_{c}}, \\
\delta \ddot{z}+n^{2} \delta z & =\frac{F_{z}}{m_{c}},
\end{aligned}
$$

where $F_{x}, F_{y}, F_{z}$ are components of the external force vector (excluding ideal gravity) resolved in Hill's frame acting on the spacecraft. In these equations, $n=\sqrt{\mu / R_{0}^{3}}$, where $R_{0}$ is the nominal orbital radius of the target space vehicle.

For two-dimensional motion, the maneuvering takes place in the $\mathrm{x}-\mathrm{y}$ plane and the $\mathrm{HCW}$ equations reduce to

$$
\begin{aligned}
\delta \ddot{x}-3 n^{2} \delta x-2 n \delta \dot{y} & =\frac{F_{x}}{m_{c}}, \\
\delta \ddot{y}+2 n \delta \dot{x} & =\frac{F_{y}}{m_{c}} .
\end{aligned}
$$

The models (12) and (13) are converted to discrete-time for the purpose of applying the approach, which has been outlined above. Assuming the sampling period, $\Delta T$, the discrete-time model has the following form

$$
x(k+1)=A x(k)+B(u(k)+w(k))
$$

where $A$ and $B$ are the discretized matrices from the HCW equations and $w(k)$ represents the disturbance. This model corresponds to the equality constraints presented in Eq. (3) as part of the set $C(x)$. Note that the formulation that uses velocity impulses as control signals is possible and leads to (14) with a different $B$ matrix; such a formulation is not pursued in this work.

\section{B. Constraints}

The set $\Omega_{i}$ of $C(x)$ in Eq. (4) is constructed given the inequality and equality constraints of the problem. Several constraints were considered in this work. During a relative motion maneuver such as docking, the space vehicle must adhere to various constraints ${ }^{11-14}$ while executing the maneuver. Depending on the portion of the relative motion maneuver being completed, different constraints must be addressed pertaining to the vehicle and the path it takes. For example, a space vehicle cannot produce limitless thrust in any direction; therefore, thrust magnitude and direction must be limited. The orientation of the vehicle is only constrained during the docking phase. For this work, a docking maneuver is studied with the Line-of-Sight (LoS) cone, thrust magnitude, and excursion constraint.

The thrust magnitude is constrained in the $x, y$, and $z$ directions for the three-dimensional case and $x$ and $y$ directions for the two-dimensional case:

$$
\begin{aligned}
& \left|u_{x}\right| \leq u_{\max } \\
& \left|u_{y}\right| \leq u_{\max } \\
& \left|u_{z}\right| \leq u_{\max }
\end{aligned}
$$

The thrust is constrained to $u_{\max }$ in each direction during flight.

A LoS cone and excursion constraint for docking are implemented. ${ }^{15}$ These constraints ensure a safe trajectory for the vehicle while avoiding collision. During the docking phase, the target vehicle's sensors constrain the approaching space vehicle to ensure it docks in the correct spot. The LoS cone constraints in three dimensions are quadratic, and are approximated using an appropriate inner approximation by polyhedral constraints

$$
A_{\text {cone }} x \leq b_{\text {cone. }}
$$

An excursion constraint is implemented to limit the vehicle in-track overshoot based on the position of the target vehicle. 


\section{Controller design}

For the two-dimensional case, a nominal LQR design is based on the state penalty matrix $Q$ set to

$$
Q=\operatorname{diag}\{100,100,1,1\}
$$

and control penalty matrix $R$ set to

$$
R=\alpha \operatorname{diag}\{1,1\}
$$

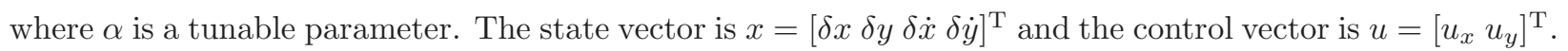
The $Q$ and $R$ matrices are constructed to put emphasis on reducing thrust and fuel consumption. The same approach is taken for the three-dimensional case with

$$
Q=\operatorname{diag}\{100,100,100,1,1,1\}
$$

and $R$ is set to

$$
R=\alpha \operatorname{diag}\{1,1,1\}
$$

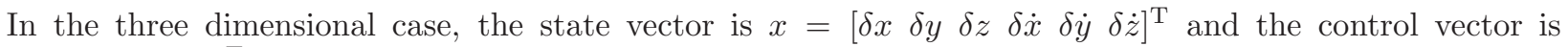
$u=\left[\begin{array}{lll}u_{x} & u_{y} & u_{z}\end{array}\right]^{\mathrm{T}}$. The control gain $K$ is determined based on the LQR procedure with the closed loop $A$ matrix defined as

$$
A_{c}=A+B K \text {. }
$$

\section{QP Problem}

Several choices of feasible sequences satisfying constraints (3) - (5) may exist. An appropriate sequence is selected by minimizing the cost function

$$
\begin{array}{ll}
\operatorname{minimize} & \sum_{k=1}^{N-1} u^{T}(k) Q_{\text {fuel }} u(k)+\Delta u^{T}(k) \Omega \Delta u(k) \\
\text { where } & \Delta u(k)=u(k+1)-u(k) \\
& \Omega=\operatorname{diag}\left\{\rho_{1}, \rho_{2}, \rho_{3}\right\}
\end{array}
$$

subject to the constraints described in Section B. In (22), $Q_{\text {fuel }}=Q_{\text {fuel }}^{T}$ is a penalty matrix, which can be increased to lower thrust levels and reduce fuel consumption. The second term in (22) penalizes the rate of change of thrust and $\Omega$ can be tuned to reduce the aggressiveness of attitude control maneuvers that need to be performed to realize the desired thrust direction. The optimization problem involved in determining the initial state and control sequences is thus a quadratic programming problem.

\section{Simulation results}

For the cases presented herein, the LoS cone constraint, thrust constraints, and rate of change of thrust are considered. The rate of the change of the thrust is controlled by augmenting a penalty to the cost function as shown in Eq. (22). Two-dimensional results are presented and then followed with three-dimensional results. The fuel results presented are determined by summing the fuel expenditure over the horizon length and not over the complete simulation. This is representative of the fuel required to get to the target set.

\section{A. Two-dimensional simulation}

First consider rendezvous maneuvering in orbital $\mathrm{x}-\mathrm{y}$ plane, where $\mathrm{x}$ corresponds to the radial direction and y corresponds to in-track direction of the Hill's frame. The origin of the Hills frame follows a circular orbit of radius of $850 \mathrm{~km}$ above the Earth, the spacecraft mass is $100 \mathrm{~kg}$ and the thrust magnitude is limited by 1.0 $\mathrm{N}$. In this implementation, the robust constrained controller governs the thrust force. The controller can be reformulated to prescribe $\Delta V$ changes by changing the discrete-time model used in the optimization. The constraints imposed on the spacecraft are to remain with the LoS cone of half angle of 5 deg and for the in-track position of the spacecraft not to fall before $0.075 \mathrm{~km}$. In addition, thrust limits were imposed and enforced during the maneuver. The horizons $\mathrm{N}=25$ and $\mathrm{N}=120$ were examined, and the sampling period was set to 2 min. 
The spacecraft is able to successfully complete a $10 \mathrm{~km}$ in-track maneuver while strictly enforcing all the imposed constraints despite a persistent level of additive acceleration disturbances, as shown in both Figures 1 and 2. The disturbance, additive to the thrust forces, has been assumed to satisfy the bounds $\|w(k)\|_{\infty} \leq 0.16 \mathrm{~N}$. Note that the disturbance size is quite large relative to the level of available thrust. Table 1 shows the $\Delta V$ expenditure for the different horizon lengths. It is evident that significantly more fuel is consumed while counteracting disturbances than when the disturbances are not present. Note that the initially optimized state and control sequences are exactly the same in the disturbance and the no disturbance cases. However, they are updated based on the feedback of the actual state and hence lead to different trajectories (always satisfying the constraints) when disturbances are and are not present.

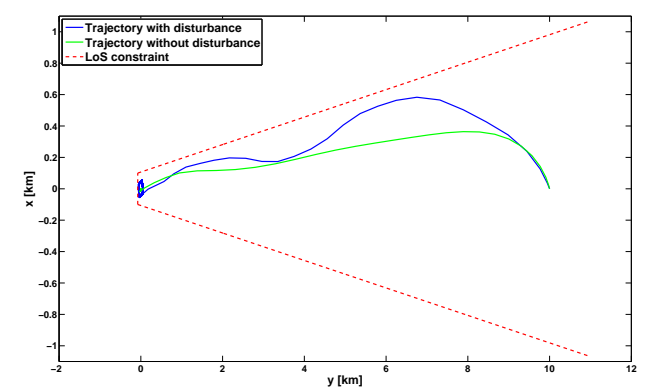

(a) Trajectory of vehicle with and without disturbance

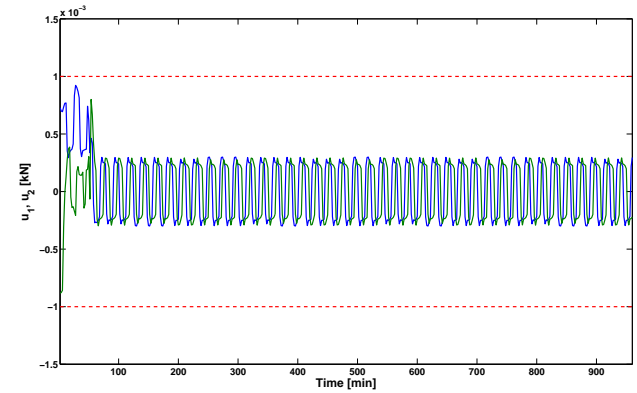

(c) Thrust along the trajectory

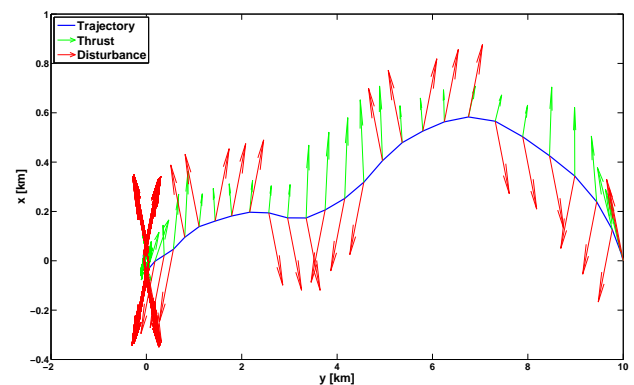

(b) Thrust and disturbance along the trajectory

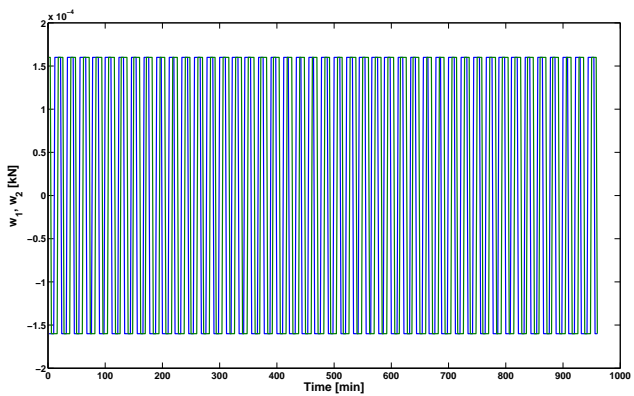

(d) Disturbance trajectory

Figure 1. The trajectory with and without disturbances for a horizon of 25 . The initial condition is $\left[\begin{array}{lllll}0 & 10 & 0\end{array}\right]^{\mathrm{T}}$.

Table 1. $\Delta V$ expenditure metric for horizons of $\mathbf{2 5}$ and $\mathbf{1 2 0}$ for the initial condition of $\left[\begin{array}{lllll}0 & 10 & 0 & 0\end{array}\right]^{\mathrm{T}}$.

\begin{tabular}{|c|c|c|}
\hline Horizon & No disturbance & Disturbance \\
\hline 25 & $0.0189 \mathrm{~km} / \mathrm{s}$ & $0.0199 \mathrm{~km} / \mathrm{s}$ \\
\hline 120 & $0.0189 \mathrm{~km} / \mathrm{s}$ & $0.0552 \mathrm{~km} / \mathrm{s}$ \\
\hline
\end{tabular}

Figures 3 and 4 show the spacecraft is able to successfully complete an off-track maneuver with imposed constraints despite a persistent level of additive disturbances. Table 2 shows that significantly more fuel is consumed while counteracting disturbances than when the disturbances are not present.

Table 2. $\Delta V$ expenditure metric for horizons of $\mathbf{2 5}$ and $\mathbf{1 2 0}$ for the initial condition of $\left[\begin{array}{lllll}-0.8 & 10 & -0.0 & 2 n\end{array}\right]^{\mathrm{T}}$.

\begin{tabular}{|c|c|c|}
\hline Horizon & No disturbance & Disturbance \\
\hline 25 & $0.0207 \mathrm{~km} / \mathrm{s}$ & $0.0213 \mathrm{~km} / \mathrm{s}$ \\
\hline 120 & $0.0227 \mathrm{~km} / \mathrm{s}$ & $0.0536 \mathrm{~km} / \mathrm{s}$ \\
\hline
\end{tabular}

The controller is able to handle disturbances for in-plane motion. By increasing the horizon the control 


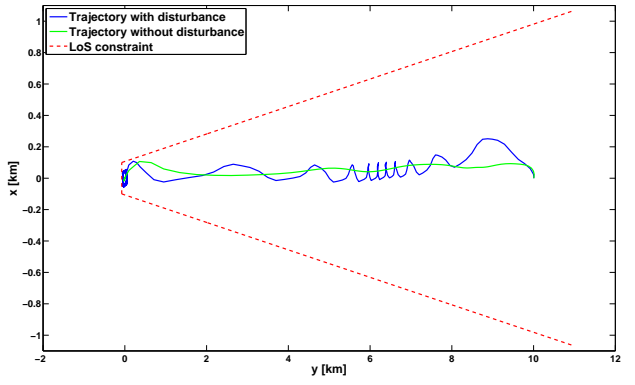

(a) Trajectory of vehicle with and without disturbance

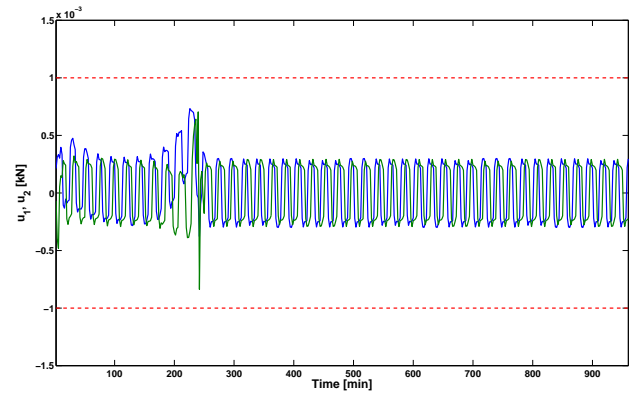

(c) Thrust along the trajectory

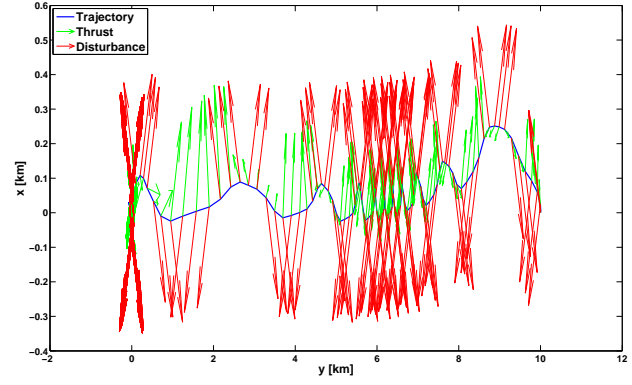

(b) Thrust and disturbance along the trajectory

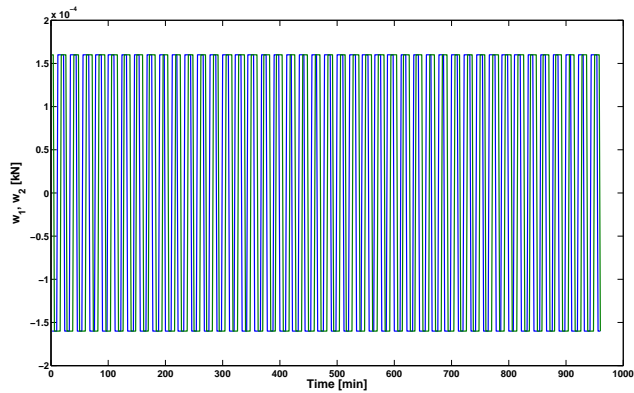

(d) Disturbance trajectory

Figure 2. The trajectory with and without disturbances for a horizon of 120 . The initial condition is $\left[\begin{array}{llllll}0 & 10 & 0\end{array}\right]^{\mathrm{T}}$.

and state sequences are determined along, the domain of recoverable initial states is increased but the initial computation time is increased with the increase of the size of the problem.

\section{B. Three-dimensional simulation}

The orbit, mass of the spacecraft, and thrust restrictions are the same in the three dimensional simulation as in the two-dimensional case. The constraints imposed on the spacecraft are to remain with the LoS cone of half angle of $5 \mathrm{deg}$ and for the in-track position of the spacecraft not to fall beyond $0.075 \mathrm{~km}$. In addition, thrust limits were imposed and enforced during the maneuver. The horizons $N=25$ and $N=120$ were examined and the sampling period was set to $2 \mathrm{~min}$. The level of disturbance was chosen to satisfy the bound $\|w(k)\|_{\infty} \leq 0.033 \mathrm{~N}$. A figure in Ref. 16 gives the order of magnitude of various perturbations for altitude varying satellite orbits. For a low earth orbit, drag has an effect on the order of .0001 N. Given the magnitude of the chosen bound on the disturbance force, it accounts for the drag force as well as other forces that may be acting on the vehicle (i.e. thruster error). The disturbance for the three dimensional cases is much smaller than the two dimensional case because the target set that satisfies all the constraints is smaller. The required minimum invariant set for the three dimensional cases must be contained within the target set, which must satisfy the constraints and therefore, is much smaller than the two dimensional cases. Hence, smaller disturbances are handled such that the state and control solutions stay within the minimum invariant set.

The spacecraft is able to successfully complete an offset $10 \mathrm{~km}$ in-track maneuver while strictly enforcing all the imposed constraints despite a persistent level of additive disturbances. See Figures 5 - 8. For the longer horizon case, the trajectory goes out before it comes back (as required by the terminal set constraint). Note that the initially optimized state and control sequences are exactly the same in the disturbance and the no disturbance cases. However, they are updated based on the feedback of the actual state and hence lead to different trajectories (always satisfying the constraints) when disturbances are and are not present. The $\Delta V$ expenditure required to complete the maneuver for each horizon is given in Table 3.

Depending on which constraints are active during a docking maneuver, the fuel consumption may vary 


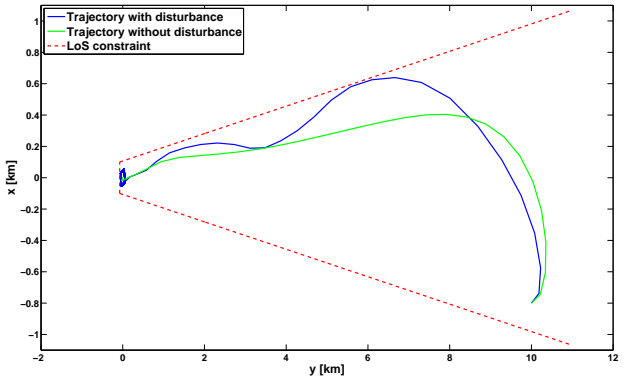

(a) Trajectory of vehicle with and without disturbance

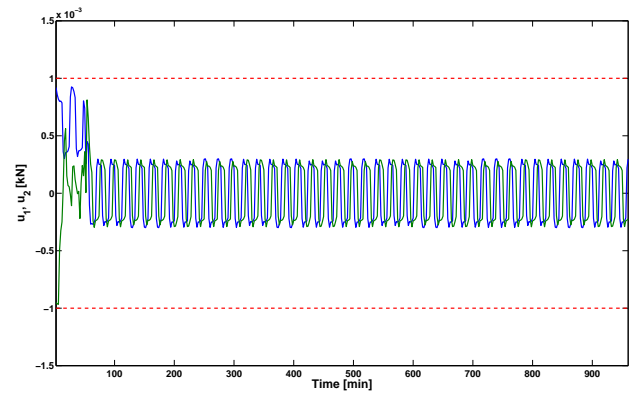

(c) Thrust along the trajectory

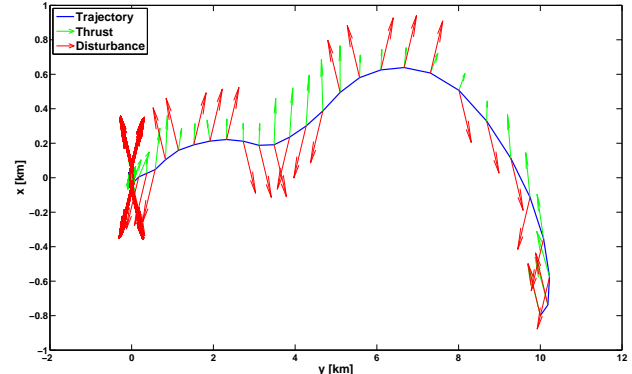

(b) Thrust and disturbance along the trajectory

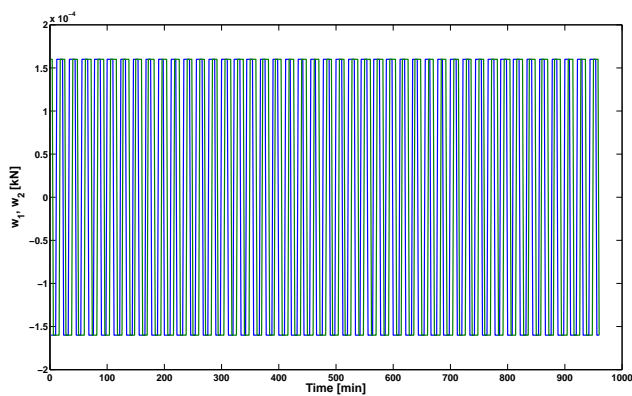

(d) Disturbance trajectory

Figure 3. The trajectory with and without disturbances for a horizon of 25. The initial condition is $[-0.810-$ $0.02 n]^{\mathrm{T}}$.

Table 3. Fuel expenditure metric for horizons of $\mathbf{2 5}$ and $\mathbf{1 2 0}$ for the initial condition of [0.6 $10-0.50 .0010 .002-$ $0.001]^{\mathrm{T}}$.

\begin{tabular}{|c|c|c|}
\hline Horizon & No disturbance & Disturbance \\
\hline 25 & $0.0227 \mathrm{~km} / \mathrm{s}$ & $0.0232 \mathrm{~km} / \mathrm{s}$ \\
\hline 120 & $0.0119 \mathrm{~km} / \mathrm{s}$ & $0.0318 \mathrm{~km} / \mathrm{s}$ \\
\hline
\end{tabular}

greatly. Three constraints were active in the above simulations: thrust magnitude costraint, excursion constraint, and a LoS cone constraint. To determine the effect of the constraints on fuel consumption, the three-dimensional case with initial condition of $\left[\begin{array}{ll}0.6 & 10-0.50 .0010 .002-0.001\end{array}\right]^{\mathrm{T}}$ is used. It is shown in Table 4 that the addition of the LoS constraints requires the most fuel to meet.

Table 4. Fuel expenditure metric for horizons of $\mathbf{2 5}$ and $\mathbf{1 2 0}$ for the initial condition of [0.6 $10-0.50 .0010 .002-$ $0.001]^{\mathrm{T}}$ for different active constraints.

\begin{tabular}{|c|c|c|c|}
\hline Horizon & Thrust & Thrust and Excursion & All constraints \\
\hline 25 & $0.0078 \mathrm{~km} / \mathrm{s}$ & $0.0080 \mathrm{~km} / \mathrm{s}$ & $.0232 \mathrm{~km} / \mathrm{s}$ \\
\hline 120 & $0.0277 \mathrm{~km} / \mathrm{s}$ & $0.0291 \mathrm{~km} / \mathrm{s}$ & $0.0318 \mathrm{~km} / \mathrm{s}$ \\
\hline
\end{tabular}

It should also be noted that the magnitude of the disturbance to be handled may be greater when the number of active constraints is decreased. For example, consider the three-dimensional case with initial condition $\left[\begin{array}{llll}0.6 & 10-0.5 & 0.001 & 0.002-0.001\end{array}\right]^{\mathrm{T}}$. Table 5 shows the maximum disturbance force the controller can compensate for. The greater the disturbance force, the greater the required fuel expenditure metric is to compensate for the disturbance. Hence, the cases with the active constraints of thrust and thrust with excursion require a greater amount of fuel to complete the required maneuver with the greater disturbance force. 


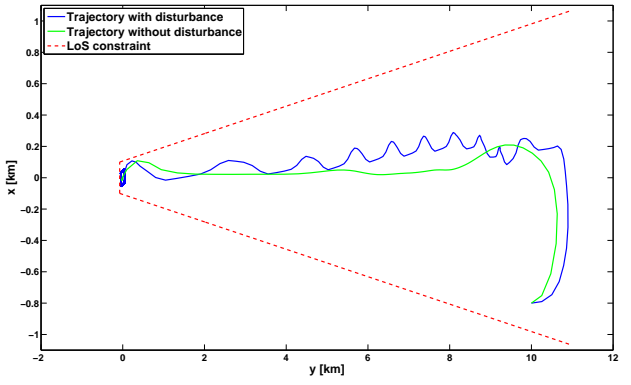

(a) Trajectory of vehicle with and without disturbance

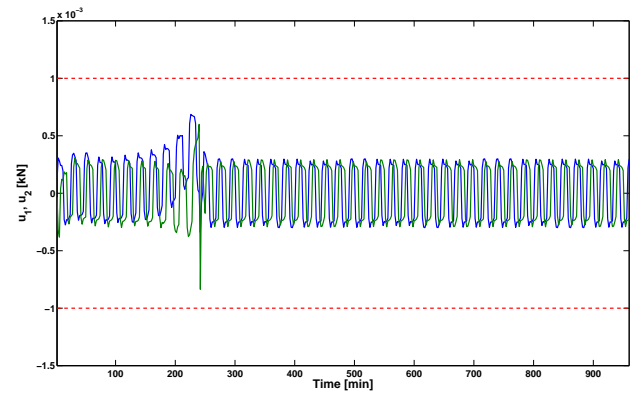

(c) Thrust along the trajectory

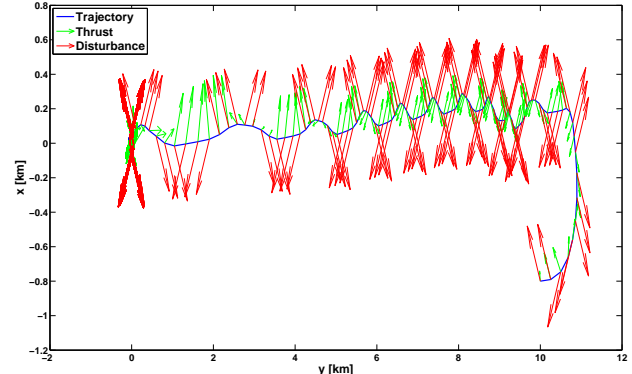

(b) Thrust and disturbance along the trajectory

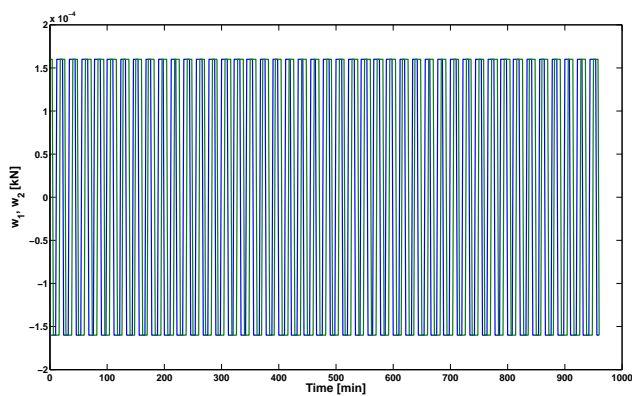

(d) Disturbance trajectory

Figure 4. The trajectory with and without disturbances for a horizon of 120 . The initial condition is $[-0.810-$ $0.02 n]^{\mathrm{T}}$.

Table 5. Maximum disturbance force and corresponding fuel expenditure metric handled by the controller

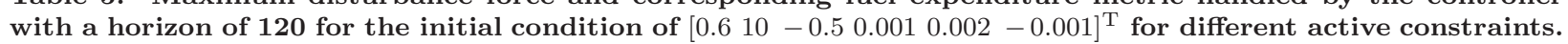

\begin{tabular}{|c|c|c|c|}
\hline Horizon & Thrust & Thrust and Excursion & All constraints \\
\hline Disturbance Force & $.140 \mathrm{~N}$ & $.0435 \mathrm{~N}$ & $0.033 \mathrm{~N}$ \\
\hline Fuel Expenditure & $0.1150 \mathrm{~km} / \mathrm{s}$ & $0.0371 \mathrm{~km} / \mathrm{s}$ & $0.0318 \mathrm{~km} / \mathrm{s}$ \\
\hline
\end{tabular}

\section{Conclusion}

A computationally fast control framework for robust spacecraft relative motion maneuvering with constraints is demostrated for two and three dimensional spacecraft relative motion. The framework presented is capable of handling bounded disturbances while guaranteeing that specified constraints are satisfied and the target set is reached for all disturbances with specified bounds. The maneuver computations reduce to solving a quadratic programming problem at the initial time instant to find a feasible maneuver path, and then generate feedback corrections along the maneuver path based on a simple constant gain linear feedback. Several numerical simulations show the effectiveness of this control framework.

\section{References} 2001.

${ }^{1}$ Show, L.-L., Juang, J.-C., and Yang, C.-D., "Nonlinear $H_{\infty}$ robust control for satellite large angle attitude maneuvers,"

${ }^{2}$ Shahid, S., Iqbal, N., and Rehan, M., "Robust Stabilization of the attitude of rigid spacecraft with bounded external disturbances using sliding mode control technique," December 2009.

${ }^{3}$ Sanyal, A., Fosbury, A., Chaturvedi, N., and Bernstein, D., "Inertia-free spacecraft attitude trajectory tracking with internal-model-based disturbance rejection and almost global stabilization," AIAA Journal of Guidance, Control, and Dynamics, Vol. 32, No. 4, 2009, pp. 1167-1178.

${ }^{4} \mathrm{Lu}, \mathrm{P}$. and Liu, X., "Autonomous trajectory planning for rendezvous and proximity operations by conic optimization," AIAA Guidance, Navigation, and Control Conference, 2012. 
${ }^{5}$ Munoz, J. and Fitz-Coy, N., "Rapid path-planning options for autonomous proximity operations of spacecraft," AIAA/AAS Astrodynamcs Specialist Conference, 2010.

${ }^{6}$ Ranieri, C., "Path-constrained trajectory optimization for proximity operations," AIAA/AAS Astrodynamcs Specialist Conference, 2008.

${ }^{7}$ Ciarcia, M. and Romano, M., "Suboptimal guidance for orbital proximity maneuver with path constraints capability," AIAA Guidance, Navigation, and Control Conference, 2012.

${ }^{8}$ Ghaemi, R., Sun, J., and Kolmanovsky, I., "Robust control of constrained linear systems with bounded disturbances with application to roll control of ships," IEEE Transactions on Automatic Control, 2012.

${ }^{9}$ Ghaemi, R., Sun, J., and Kolmanovsky, I., "Robust control of ship fin stabilizers subject to disturbances and constraints," Proceedings of American Control Conference, 2009.

${ }^{10}$ Wie, B., Space Vehicle Dynamics and Control, AIAA Education Series, Virginia, 2008.

${ }^{11}$ Park, H., Cairano, S. D., and Kolmanovsky, I., "Model Predictive Control for spacecraft rendezvous and docking with a rotating/tumbling platform and for debris avoidance," Proceedings of American Control Conference, 2011.

${ }^{12}$ Park, H., Cairano, S. D., and Kolmanovsky, I., "Linear quadratic Model Predictive Control approach to spacecraft rendezvous and docking," Proceedings of 21st AAS/AAIA Space Flight Mechnics Meeting, 2011.

${ }^{13}$ Park, H., Cairano, S. D., and Kolmanovsky, I., "Model Predictive Control of spacecraft docking with a non-rotating platform," Proceedings of the 18th IFAC World Congress, 2011.

${ }^{14}$ Weiss, A., Kolmanovsky, I., Baldwin, M., and Erwin, R., "Model predictive control of three dimensional spacecraft rendezvous and proximity maneuvering," Proceedings of American Control Conference, 2012.

${ }^{15}$ Richards, A. and How, J., "Performance evaluation of rendezvous using model predictive control," AIAA Guidance, Navigation, and Control Conference and Exhibit, 2003.

${ }^{16}$ Montenbruck, O. and Gill, E., Satellite Orbits: Models, Methods, and Applications, Springer-Verlag, New York, NY, 2000, pp. 24-32, 233-253. 

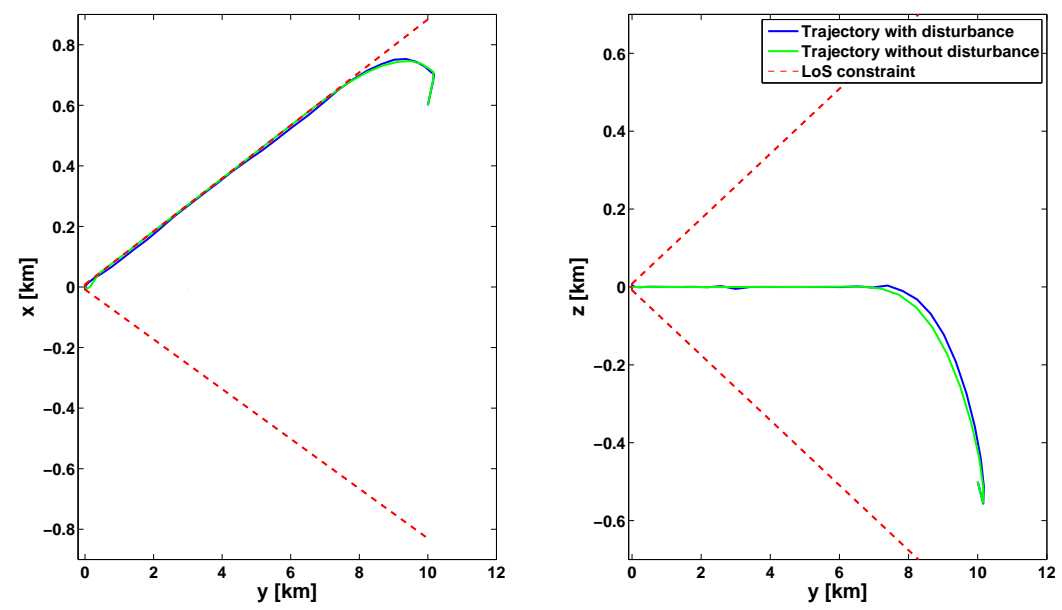

(a) Planar trajectory in the $x-y$ and $z-y$ plane

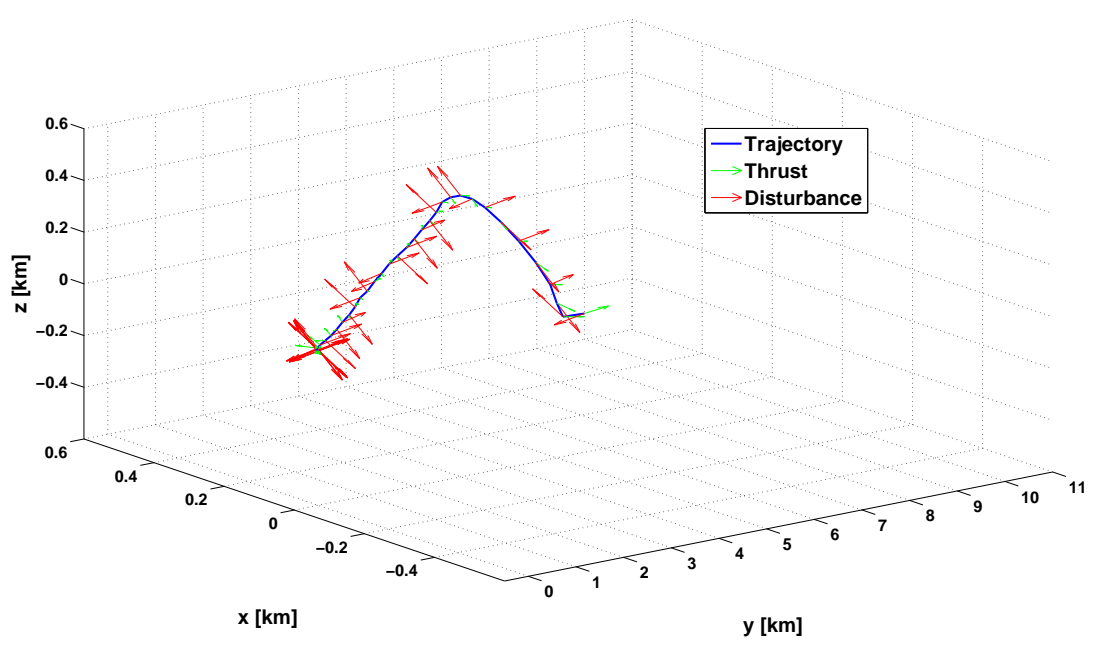

(b) Thrust and disturbance along the trajectory

Figure 5. The trajectory with and without disturbances for a horizon of 25. The initial condition is $[0.610-$ $0.50 .0010 .002-0.001]^{\mathrm{T}}$. 


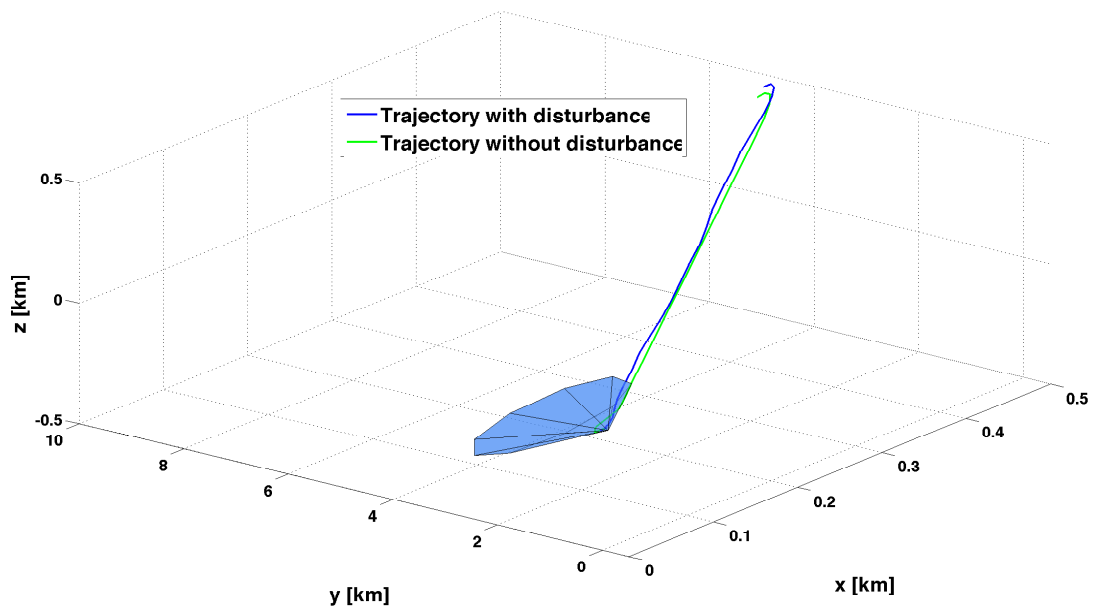

(a) Three dimensional trajectory

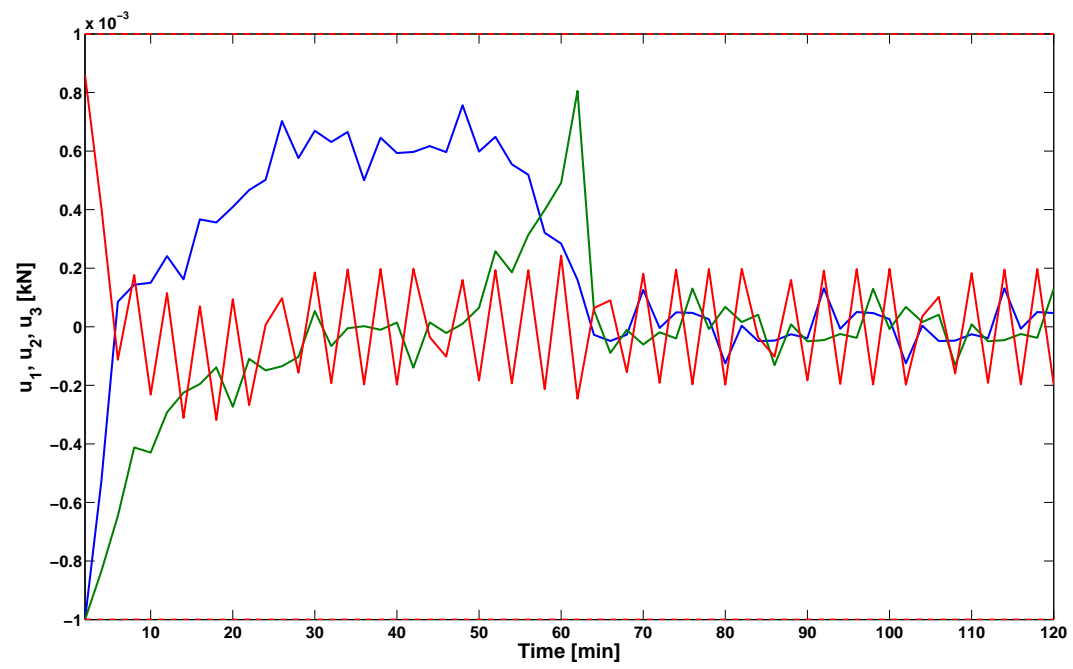

(b) Thrust along the trajectory

Figure 6. The three-dimensional trajectory with and without disturbances for a horizon of 25 and the thrust along the trajectory. The initial condition is $\left[\begin{array}{llllll}0.6 & 10 & -0.5 & 0.001 & 0.002 & -0.001\end{array}\right]^{\mathrm{T}}$. 

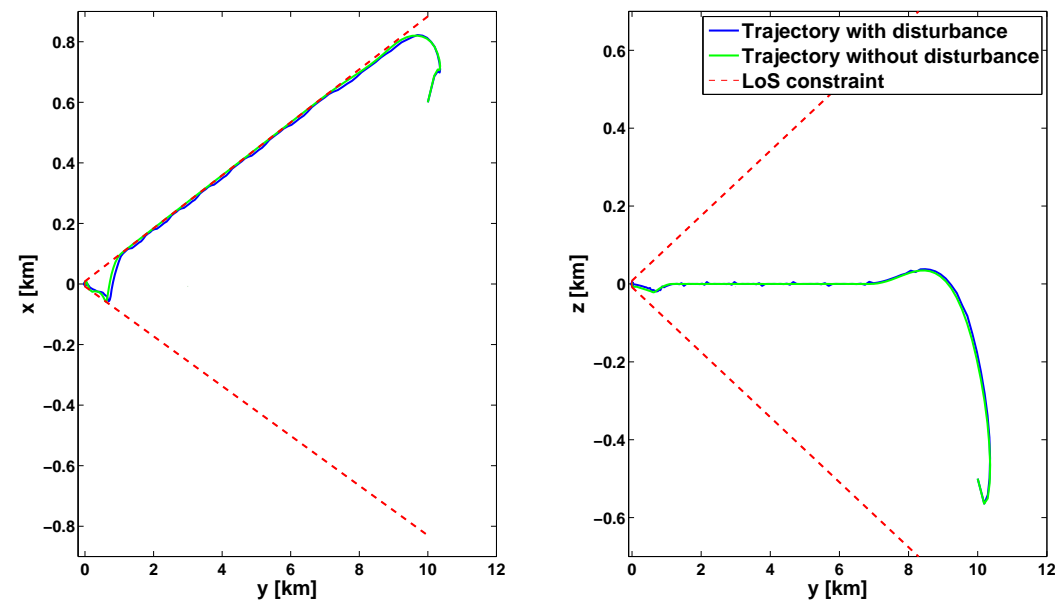

(a) Planar trajectory in the $x-y$ and $z-y$ plane

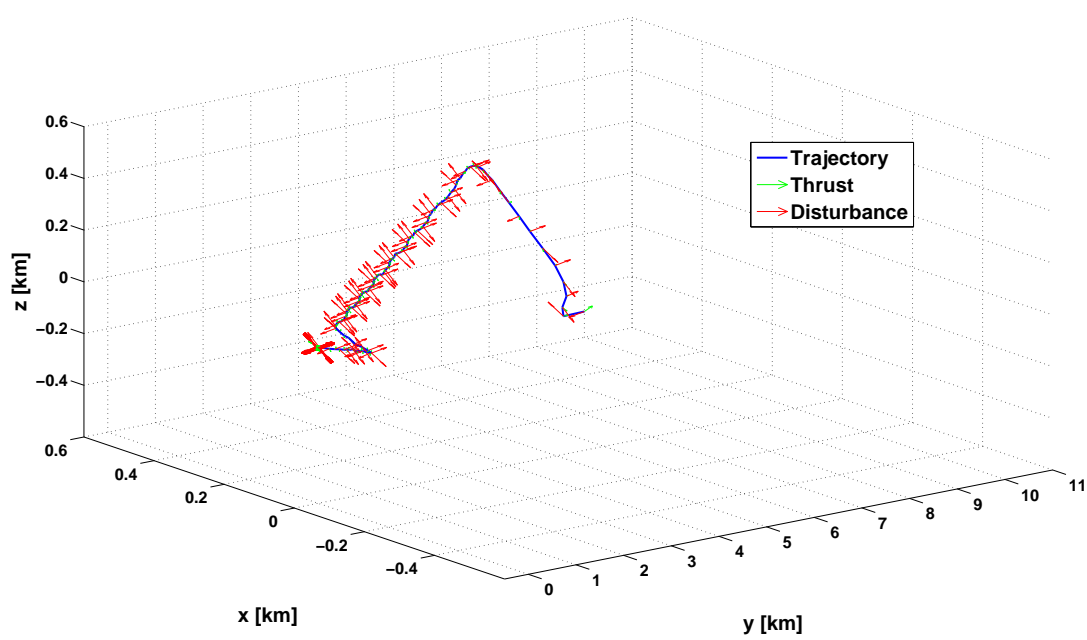

(b) Thrust and disturbance along the trajectory

Figure 7. The trajectory with and without disturbances for a horizon of 120 . The initial condition is $[0.610-$ $0.50 .0010 .002-0.001]^{\mathrm{T}}$. 


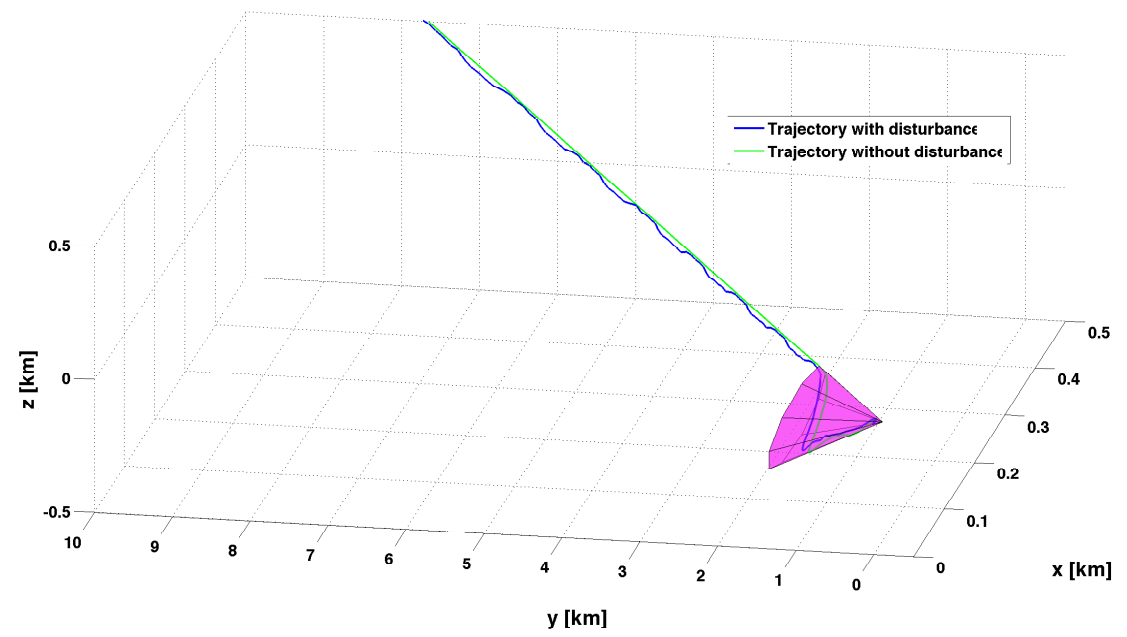

(a) Three dimensional trajectory

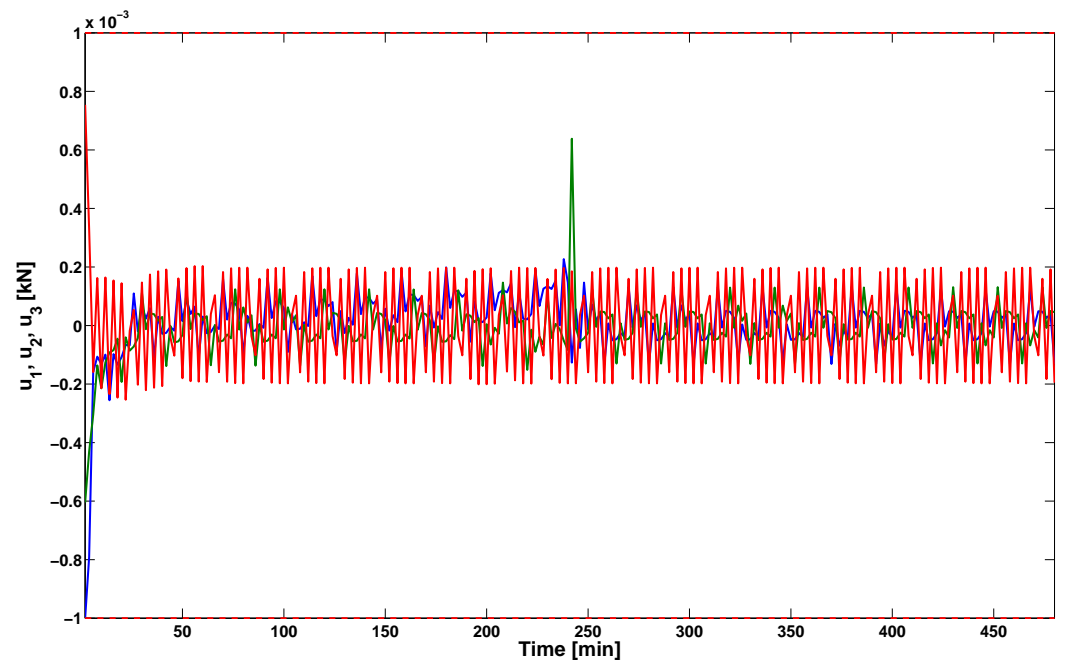

(b) Thrust along the trajectory

Figure 8. The three-dimensional trajectory with and without disturbances for a horizon of 120 and the thrust

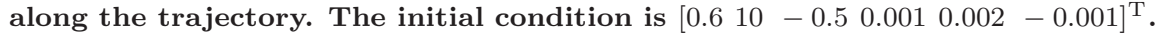

